\title{
Intracelluar pH (pHi) Measurements in the In Vitro Tadpole Brainstem: Direct Correlations between Changes in pHi and Ventilation
}

\author{
Debora A. Zamora ${ }^{1}$, C.R. Marutha Ravindran ${ }^{1}$, James N. Bayne ${ }^{1}$, James C. Leiter ${ }^{2}$ and Matthew J. \\ Gdovin $^{1, *}$ \\ ${ }^{1}$ Department of Biology, TheUniversity of Texas at San Antonio, 1 UTSA Circle, San Antonio, TX 78249, USA \\ ${ }^{2}$ Department of Physiology, Dartmouth Medical School, HB 7700, Hanover NH 03755, Germony
}

\begin{abstract}
Central respiratory chemoreceptors measure $\mathrm{pH}$ in the brain stem and are an integral part of the neural circuitry that modulates respiratory rhythmogenesis, specifically in response to hypercapnic acidosis. Central respiratory chemoreceptor membrane potential and/or action potential firing rate are altered in response to changes in intracellular $\mathrm{pH}(\mathrm{pHi})$, which changes with the hydration of $\mathrm{CO}_{2}$ in both the extracellular and intracellular space, however the role of cellular changes in chemoreceptor properties on respiratory motor output has been difficult to identify. We studied whole nerve respiratory activity while simultaneously visualizing $\mathrm{pHi}$ dynamics using the $\mathrm{pH}$-sensitive dye, BCECF, in the spontaneously active in vitro tadpole brainstem. The isolated, superfused tadpole brainstem is well oxygenated and retains synaptic connectivity among respiratory central pattern generators, central respiratory chemoreceptors, and respiratory motor neuronsunder physiological conditions, where mammalian preparations do not. An ammonium prepulse was used to selectively induce a decrease in pHi. Our results show intracellular $\mathrm{pH}$ is regulated differently in cells located in chemosensitive and non-chemosensitive regions of the tadpole brainstem during hypercapnia. We were also able to show an inverse correlation between $\mathrm{pHi}$ in cells located in chemosensitive regions of the tadpole brainstem and whole nerve respiratoryrelated activity. Using this approach, the microenvironment of individual cells may be manipulated while monitoring real time changes in $\mathrm{pHi}$, neuronal activity and ventilatory-related activity to elucidate the role of a variety of signals in eliciting changes in ventilation.
\end{abstract}

Keywords: Central respiratory chemoreceptors, respiration, carbon dioxide, $\mathrm{pH}$.

\section{INTRODUCTION}

The characterization of the cellular mechanisms involved in $\mathrm{CO}_{2} / \mathrm{H}^{+}$chemoreception has been the focus of recent research $[1,2]$. Unequivocally identifying a chemosensitive cell as being respiratory in nature continues to be problematic when studying central respiratory chemoreception. It is difficult to establish firm causal relationships between stimulus, effector elements and responses in intact preparations because each neuron makes only a small contribution to the overall response - different patterns of neuronal activity can only be correlated with particular responses. In reduced preparations, causal links may be established between sequential neural elements, but the biological importance of any particular response is moot since the neural activities are frequently unconnected to meaningful physiological responses. Brain areas involved in respiratory chemosensitivity have been identified by the discrete application of $\mathrm{CO}_{2} / \mathrm{H}^{+}$ $[3,4,5]$ and differences in rates of $\mathrm{pHi}$ regulation between putative chemosensitive and non-chemosensitive cells have

*Address correspondence to this author at the Department of Biology, The University of Texas at San Antonio, 1 UTSA Circle, San Antonio, Texas 78249, USA; Tel: 210-458-4275; Fax: 210-458-5658;

E-mail: matthew.gdovin@utsa.edu been proposed [6]. In addition, separate studies using $\mathrm{pH}-$ sensitive dyes and whole cell recordings have found significant correlations between pHi and neuronal firing rate [1]. Moreover, lesion studies in which particular classes of neurons are killed, knocked out or inhibited have identified particular neuronal populations in specific regions of the brain that contribute to $\mathrm{CO}_{2}$ chemosensitivity, but these studies do not reveal the positive functional aspects of these neurons (i.e., how does the neuronal activity of these neurons mediate chemosensitivity) since the neurons of interest are absent from the animal or preparation [7-9]. While these studies provide valuable information regarding the cellular effects of $\mathrm{CO}_{2} / \mathrm{H}^{+}$and the neuronal populations responsible for central $\mathrm{CO}_{2}$ chemosensitivity, it has not been possible to attribute changes in respiratory motor output to the specific pattern of neuronal activity during changes in $\mathrm{CO}_{2}$ or $\mathrm{pH}$ in specific neurons. Addressing this issue requires simultaneous measurements of pHi dynamics and whole nerve respiratory output in a single preparation. The development of the in vitro tadpole brainstem preparation and simultaneous measurement of pHi using 2',7'-bis-(2-carboxyethyl)-5-(and-6)carboxyfluorescein, acetoxymethyl ester (BCECF, AM) provides a novel model to determine if there is a relationship between changes in pHi in specific cells and neural activity related to ventilation. 
Here we describe a method to simultaneously measure pHi dynamics in small numbers of neurons located in known chemosensitive brainstem regions and whole nerve activity related to the respiratory output in a reduced amphibian preparation, the in vitro tadpole brainstem of the larval Lithobatescatesbeianus, formerly Rana catesbeiana. Recent development of neonatal and adult rodent in vitro, en bloc, and in situ preparations have provided valuable information regarding the neural substrates of respiratory rhythmogenesis [10-15]. Concerns regarding the interpretation of use of these preparations are valid and have been reported $[14,16]$. One concern centers around the "respiratory-like" motor pattern recorded from these preparations, as they do not produce the augmenting inspiratory and declining post-inspiratory patterns recorded in vivo[14], but instead demonstrate a rapid onset burst pattern similar to gasping [17]. In addition, these preparations are conducted in conditions that do not mimic physiological conditions in vivo; supraphysiological bath $\mathrm{K}^{+}$ and reductions in bath temperature as low as $25^{\circ} \mathrm{C}$ produce a preparation with a hypoxic, hyperkalemic, acidic core [1819]. Attempting to use these preparations to study central respiratory chemoreception is limited, as "metabolic conditions such as hypercapnia and hypoxia in the deeper brainstem tissue may impair network connectivity and synaptic function, resulting in either a 2- or 1 phase pattern" rather than the 3-phase rhythm associated with eupnea [20]. Smith et al., [20] reported a switch from the eupnic 3-phase pattern to to a 1 phase pattern in response to changes in $\mathrm{CO}_{2}$ in the in situ perfused rat brainstem-spinal cord preparation. The tadpole brainstem of Lithobates catesbeianus is well oxygenated [21], retains the circuitry necessary to produce spontaneous respiratory output and has a measurable respiratory response to changes in $\mathrm{CO}_{2}$ [22-23]. For these reasons, the ability to monitor whole nerve respiratory activity and changes in pHi simultaneously provides a powerful tool to dissect the mechanistic connections among the neural elements involved in respiratory responses to $\mathrm{CO}_{2}$.

\section{METHODS}

\section{In Vitro Brainstem Preparation}

\section{Animals}

Experiments were performed on Lithobates catesbeianus tadpoles (BCECF calibration $n=57$; Hypercapnic Acidosis n = 55) of developmental stages 3-20 [24] of either sex obtained from a commercial supplier (Sullivan, TN USA). All tadpoles were housed in aquaria and were maintained at 24 $26^{\circ} \mathrm{C}$ with a $12 \mathrm{~h}: 12 \mathrm{~h}$ light:dark photoperiod. Tadpoles were fed on a daily basis (Fish Flakes, Wardly) as needed so that food was not a limiting resource. Animal care and experimental protocols were approved by the University of Texas at San Antonio Institutional Animal Care and Use Committee.

\section{Dissection}

Tadpoles were anesthetized in 3-aminobenzoic acid ethyl ester (MS 222; Sigma-Aldrich, St. Louis, MO) dissolved in distilled water $(1: 10,000)$ until unresponsive to tail pinch and weighed. Using a dissecting microscope, the dorsal cranium was removed, and the cranial and spinal nerves were severed at their ostia. After the dura and arachnoid were removed dorsally and ventrally, the brainstem was transected just caudal to the level of the second spinal (hypoglossal) nerve root and just rostral to cranial nerve V. Throughout the dissection, the brainstem was continuously superfused with artificial cerebrospinal fluid (aCSF) bubbled with $\mathrm{O}_{2}$. The composition of the aCSF was as follows (in $\mathrm{mM}$ ): $\mathrm{NaCl} 104$; $\mathrm{KCl}$ 4.0; $\mathrm{MgCl}_{2}$ 1.4; D-glucose 10; $\mathrm{NaHCO}_{3} 25$; and $\mathrm{CaCl}_{2}$ 2.4. Following dissection, the brainstem was incubated in oxygenated 2',7'-bis-(2-carboxyethyl)-5-(and-6)-carboxyfluorescein, acetoxymethyl ester (BCECF-AM; Molecular Probes, Eugene, Oregon) .

\section{Recording Chamber}

The brainstem was removed as described above then transferred to a superfusion recording chamber maintained at room temperature, $21-23{ }^{\circ} \mathrm{C}$. The brainstem was secured ventral side up to the floor of the chamber using a stainless steel anchor with Lycra ${ }^{\circledR}$ threads $0.1 \mathrm{~mm}$ thick and $2.0 \mathrm{~mm}$ apart (SHD-26GKIT, Warner Instrument Corporation). The bottom of the chamber was constructed from a $22 \times 40 \mathrm{~mm}$ glass cover slip with a thickness of $0.13-0.17 \mathrm{~mm}$ and had a working volume of $180 \mu \mathrm{l}$. The brainstem was superfused with aCSF that had been equilibrated with a $\mathrm{CO}_{2} / \mathrm{O}_{2}$ gas mixture in an external tonometer at room temperature. The superfusate entered the recording chamber through an inflow aperture at a rate of $2 \mathrm{ml} / \mathrm{min}$ and exited from the opposite end via a vacuum. The aCSF $\mathrm{pH}$ was measured in the tonometer (Orion, 420A) and bath (Orion 9863BN), and aCSF $\mathrm{pH}$ was adjusted by changing the amount of $\mathrm{CO}_{2}$ bubbling into the tonometer.

\section{Whole Nerve Recordings}

Neural recordings of fictive gill and lung ventilation were obtained from the roots of CN VII using glass suction electrodes. Suction electrodes were made from thin walled borosilicate glass capillaries (O.D. $1 \mathrm{~mm}$; ID $0.5 \mathrm{~mm}$ ), pulled to a fine tip with a horizontal micropipette puller (Flaming/Brown, P-97). Pipettes were cut with a diamond pen and smoothed by heating on an open flame to achieve inner tip diameters that fit the cranial nerve of interest (approximately 220 to $315 \mu \mathrm{m}$ ). A $0.25 \mathrm{~mm}$ diameter silver grounding wire was wrapped around the outside of the pipette down to the tip of the electrode. Suction electrodes were filled with filtered aCSF solution described above. Efferent neural activity was amplified (10,000X; AM Systems, 1700), filtered (10 $\mathrm{Hz}$ to $500 \mathrm{~Hz}$ ), simultaneously averaged with a moving time averager (CWE, MA-821) and digitized and recorded on a Pentium 4 PC (Datapac 2000 software).

\section{Fluorescence Imaging}

BCECF is a dual excitation ratio metric dye that when excited at $440 \mathrm{~nm}$ (its iso-excitation point) is $\mathrm{pH}$ insensitive and when excited at $495 \mathrm{~nm}$ is $\mathrm{pH}$-sensitive [25]. BCECF reports only cytoplasmic changes in $\mathrm{pH}$ and allows rapid kinetic changes of $\mathrm{pH}$ as small as 0.01 to be monitored [2628.] The addition of acetoxymethyl (-AM) makes BCECF temporarily membrane permeable and therefore provides a non-invasive methods of dye loading [26-28.] Once inside the cell, hydrolysis of the acetyl ester linkage by enzymatic cleavage regenerates the less permeable original compound [26]. BCECF has a slow rate of dye leakage and does not appear to be harmful to cells [26]. 


\section{Dye Preparation}

BCECF-AM stock 0.004 M (Molecular probes, Eugene, OR) was made by dissolving $1 \mathrm{mg}$ BCECF-AM in methylsulfoxide (DMSO) $(201 \mu \mathrm{L})$. Fifty $\mu \mathrm{L}$ of each BCECF stock solution was added to $10 \mathrm{ml}$ aCSF for a final BCECF concentration of $40 \mu \mathrm{M}$.

\section{Dye Incubation}

The in vitro tadpole brainstem was incubated in $10 \mathrm{ml}$ of $40 \mu \mathrm{M}$ BCECF, prepared as described above, for 30 minutes in the dark. Following dye incubation, the brainstem was washed for 30 minutes in oxygenated aCSF.

\section{Imaging of BCECF-loaded Neurons}

The superfusion chamber containing the brainstem was placed under an upright microscope (ECLIPSE E600FN Nikon, Melville, NY) mounted with a charge-coupled device (CCD) camera (MicroMAX, Roper Scientific, Trenton, NJ) connected to a Pentium computer (Hewlett Packard Company, Palo Alto, CA). Neurons were excited for 200 - 400 msec with light from a $175-\mathrm{W}$ xenon arc lamp (Sutter Instrument, Novato, CA) that was filtered to 495 or $440 \mathrm{~nm}$ using a high speed filter changer (Lambda DG-4, Sutter Instrument, Novato, CA). Emitted light passed through a dichroic mirror with a high pass cut off of $515 \mathrm{~nm}$ and a $535 \pm$ 25 nm emission filter (Chroma Technology, Brattleboro, VT). Data were collected with an image acquisition software program (MetaMorph/MetaFluor, Universal Imaging Corporation, Downingtown, PA) for later analysis.

\section{BCECF Calibration}

The calibration solution contained in $(\mathrm{mM})$ : $\mathrm{KCl} 104$; $\mathrm{CaCl}_{2}$ 2.4; $\mathrm{MgCl}_{2}$ 1.4; N-methyl-D-glucamine (NMDG)-N2-hydroxyethylpiperazine-N'-2-ethanesulfonic acid (HEPES); D-glucose 10; Sucrose 25; and 0.016 nigericin titrated with either $\mathrm{KOH}$ or $\mathrm{HCl}$ to desired $\mathrm{pH}$. All calibration points were normalized to $\mathrm{pH} 7.2$ and a calibration curve ranging from $\mathrm{pH} 6.2$ to 8.6 was constructed.

\section{One-Point Nigericin Technique}

Brainstems from subsequent experiments were superfused with high $\mathrm{K}^{+}$/nigericin solution set at $\mathrm{pH} 7.2$ at the end of each experiment. Once fluorescence acquisitions reached steady state an averaged nigericin response was acquired to normalize experimental fluorescence ratios (495/440). Normalized fluorescence was converted into pHi values using the equation obtained from the above mentioned calibration curve.

\section{Hypercapnic Acidosis}

Intracellular ( $\mathrm{n}=55)$ emitted BCECF fluorescence during normocapnic (bath $\mathrm{pH}=7.8$ ) and hypercapnic (bath $\mathrm{pH}=$ 7.4) bath conditions was recorded in cells located in chemosensitive and non-chemosensitive regions of the spontaneously rhythmic in vitro tadpole brainstem preparation. Briefly, the brainstem was dissected and incubated in BCECF $(40 \mu \mathrm{M})$ for 30 minutes as described above. Following the 30 minute aCSF wash, the brainstem was transferred to a superfusion chamber (RC-26GLP, Warner Instrument Corporation) for optical recordings of intracellular fluorescence. The superfusate was equilibrated in external tonome- ters with an $\mathrm{O}_{2} / \mathrm{CO}_{2}$ gas mixture to desired $\mathrm{pH}$ values and had a flow rate of $2 \mathrm{ml} / \mathrm{min}$. The brainstem was superfused with control aCSF (pH7.8) for at least 10 minutes and baseline fluorescence was acquired. The $\mathrm{PCO}_{2}$ was then raised to attain a $\mathrm{pH}$ value of 7.4 and after a 10 minute equilibration period, fluorescence acquisitions were taken every minute for 10 minutes. Baseline fluorescence ( $\mathrm{pH} 7.8)$ post hypercapnic exposure was attained and after a 10 minute equilibration period acquisitions were taken every minute for $10 \mathrm{~min}$ utes.

A subset of animals of developmental stages 3-10 ( $\mathrm{n}=$ 10) and 11-20 $(n=8)$ were used to describe developmental changes in respiratory activity during hypercapnic acidosis, while simultaneously collecting optical recordings of $\mathrm{pHi}$. Respiratory variables, mentioned above in Respiratory Variables, were acquired and analyzed using Datapac 2000 software. Experimental time included five experimental conditions: control (C1), transition to hypercapnic acidosis (T1), hypercapnic acidosis (HA), transition back to control (T2), and final control (C2). Gill and lung activity were analyzed separately. For statistical analyses, data were normalized to a percent change from the initial control (C1) value.

\section{Statistical Analysis}

Since each animal experienced all five experimental periods, Repeated Measure (RM) One-way ANOVAs were run for each respiratory variable measured. If data did not meet the assumptions of parametric statistical tests, then a nonparametric RM Mann-Whitney Rank Sum Test was used. $P$ values $<0.05$ were considered significant. Unless otherwise stated, values reported are mean \pm one standard error of the mean (SEM).

\section{pHi Regulation in Chemosensitive and Non-chemosensitive Regions}

pHi regulation of cells in chemosensitive $(n=35)$ and non-chemosensitive $(n=22)$ regions of the tadpole brainstem was determined using the fluorescence dye, BCECF. Briefly, BCECF loaded cells were exposed to hypercapnic acidosis and fluorescence acquisition were acquired every 15 seconds for the initial 5 minutes of hypercapnic exposure followed by acquisitions every 30 seconds for an additional 10 minutes. To determine if $\mathrm{pHi}$ regulation occurred, linear regressions were done from the lowest $\mathrm{pHi}$ value observed during hypercapnic acidosis to the average baseline hypercapnic response, the last 5 minutes of hypercapnic exposure.

Ammonia prepulse was used $(n=4)$ to determine if cells that lacked $\mathrm{pHi}$ regulation during hypercapnic acidosis were able to exhibit regulation during intracellular acidification at a constant pHo. Briefly, BCECF fluorescence was obtained in cells that were exposed to hypercapnic acidosis $\mathrm{pH} 7.4$ and control solution $\mathrm{pH}$ 7.8. The brainstem was then superfused with $\mathrm{NH}_{4} \mathrm{Cl}$ until the characteristic alkalinization plateau was reached and started to decline, approximately 2 minutes. Upon exposure and removal of $\mathrm{NH}_{4} \mathrm{Cl}$, fluorescence acquisitions were taken every 15 seconds to monitor the changes in intracellular $\mathrm{pH}$.

\section{Statistical Analysis}

A t-test was run to compare pHi regulation rates from cells located in chemosensitive and non-chemosensitive re- 
gions of the tadpole brainstem. A t-test was run to compare pHi regulation rates during two different methods of intracellular acidification, hypercapnic acidosis and $\mathrm{NH}_{4} \mathrm{Cl}$ prepulse. A t-test was run to compare levels of intracellular acidification during hypercapnic acidosis and $\mathrm{NH}_{4} \mathrm{Cl}$.

\section{RESULTS}

\section{pHi Regulation in Chemosensitive and Non-chemosen- sitive Regions}

The rate of $\mathrm{pHi}$ regulation during hypercapnic acidosis was significantly $(\mathrm{P}<0.05)$ greater in cells located in nonchemosensitive regions $(n=22 ; 0.06 \pm 0.14 \mathrm{pH}$ unit change $\left.\min ^{-1}\right)$ than cells located in chemosensitive regions $(n=34$; $0.01 \pm 0.01 \mathrm{pH}$ unit change $\mathrm{min}^{-1}$ ) (Fig. 1). With respect to the presence of $\mathrm{pHi}$ regulation, there was a mixed population of cells within both non-chemosensitive and chemosensitive regions of the brainstem in that not all cells demonstrated pHi regulation. However, a larger percentage of cells within the non-chemosensitive region (76\%) exhibited pHi regulation during hypercapnic acidosis compared to cells within chemosensitive regions (47\%).
To determine if cells that lacked pHi regulation during hypercapnic acidosis exhibited the ability to regulate intracellular $\mathrm{pH}$, a subset of cells $(\mathrm{n}=4)$ from a chemosensitive region that did not exhibit $\mathrm{pHi}$ regulation during hypercapnic acidosis, were exposed to an ammonia prepulse (60 mM $\mathrm{NH}_{4} \mathrm{Cl}$ was added to the perfusion bath for five minutes), an experimental treatment that causes an initial alkalosis followed by plateau alkalinization as $\mathrm{NH}_{4}^{+}$enters the cells (probably through potassium channels), but causes a decrease in $\mathrm{pHi}$ at a constant $\mathrm{pHo}$ when $\mathrm{NH}_{4} \mathrm{Cl}$ is removed from the perfusate and the intracellular $\mathrm{NH}_{4}{ }^{+}$sheds a proton and leaves the cell as $\mathrm{NH}_{3}$. Although there was a similar drop in pHi during hypercapnic acidosis and the ammonia prepulse, the rate of $\mathrm{pHi}$ regulation was significantly $(\mathrm{P}<0.05)$ greater following the ammonia prepulse induced intracellular acidification $(0.015 \pm 0.003 \mathrm{pH}$ unit change $\mathrm{min}^{-1}$ ) than during intracellular acidification associated with hypercapnic acidosis $\left(0.003 \pm 0.002 \mathrm{pH}\right.$ unit change $\left.\min ^{-1}\right)$. An example of the pHi profile recorded from a single cell located in a chemosensitive region exposed to an ammonia prepulse is shown in Fig. (2).

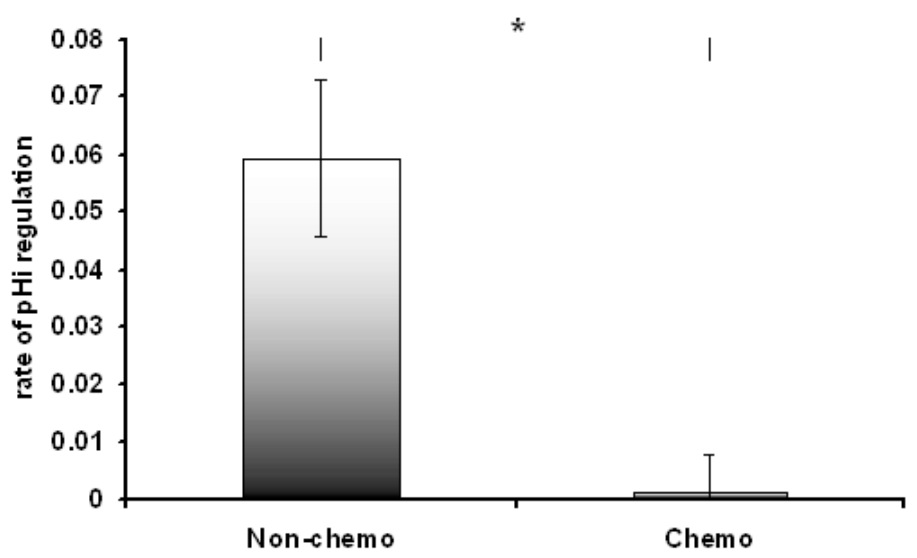

Fig. (1). pHi regulation in non-chemosensitive $(n=22)$ and chemosensitive $(n=34)$ regions of the tadpole brainstem during hypercapnic acidosis. pHi regulation ( $\mathrm{pH}$ unit change $\mathrm{min}^{-1}$ ) of cells located in non-chemosensitive regions $(0.06 \pm 0.14)$ was significantly* $(\mathrm{P}<0.05)$ greater than $\mathrm{pHi}$ regulation of cells located in chemosensitive regions $(0.01 \pm 0.01)$.

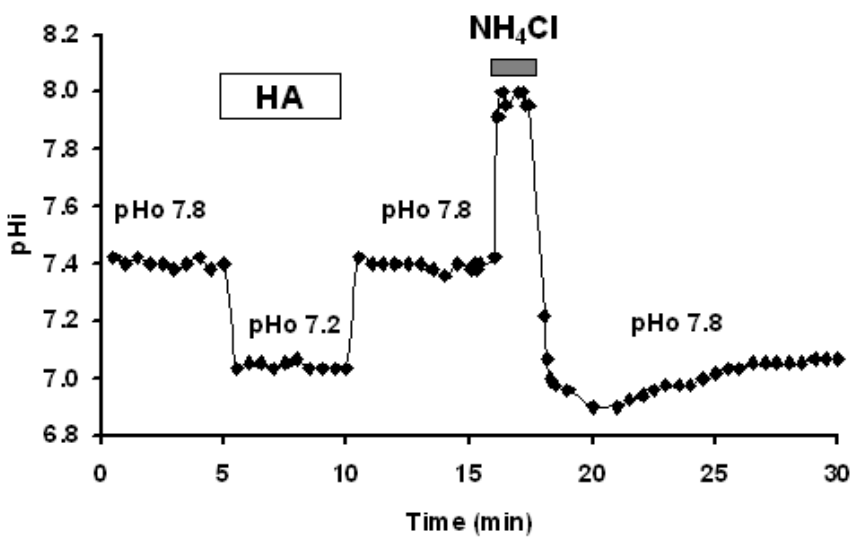

Fig. (2). pHi profile of a chemosensitive cell during control $\mathrm{pH}$, hypercapnic acidosis, return to control, and $\mathrm{NH}_{4} \mathrm{Cl} \mathrm{prepulse}^{\mathrm{Note}}$ that there is no $\mathrm{pHi}$ regulation following hypercapnic acidosis, but the same cell regulates $\mathrm{pHi}$ following intracellular acidification with $\mathrm{NH}_{4} \mathrm{Cl}$ prepulse. 
Correlation of pHi and Whole Nerve Respiratory Activity

\section{Whole Nerve Activity}

To describe the relationship between whole nerve respiratory activity during the transition to higher levels of $\mathrm{CO}_{2}$ and pHi of cells located in documented chemosensory and non-chemosensory regions, we recorded respiratory activity from cranial nerve VII in tadpoles at Taylor and Kollros [24] developmental stages 3-10 $(n=10)$ and 11-20 $(n=8)$. Two distinct, developmentally-dependent respiratory patterns are observed in vivo [29] and may be recorded from respiratoryrelated cranial nerves in vitro: a relatively high frequency, low amplitude pattern corresponding to gill ventilation, and a low frequency, high amplitude pattern corresponding to lung ventilation [30-32]. To characterize the hypercapnic respiratory response, we measured both the lung burst frequency, cycle length, burst duration, inter-burst interval (IBI), and the amplitude of rectified, integrated nerve activity and the gill burst frequency, cycle length, burst duration, inter-burst interval (IBI), and amplitude. Experimental recording time included five experimental periods: control (C1), transition to hypercapnic acidosis (T1), hypercapnic acidosis (HA), transition back to control (T2), and final control (C2).

\section{Gill Respiratory Variables - Early Developmental Stages}

All preparations exhibited the expected high frequency, small amplitude fictive gill bursting pattern. In early stage developmental tadpoles, the gill respiratory values during superfusion with control aCSF were (in seconds): cycle time $1.54 \pm 0.23$, burst duration $1.15 \pm 0.10$, and IBI $0.39 \pm 0.18$. There were no significant differences in gill burst frequency, cycle (Fig. 3), duration, and IBI in response to the exposure to hypercapnic acidosis in the early stage (Table 1). Gill burst amplitude measured in arbitrary units (au) did not change significantly throughout the different experimental conditions.

\section{Gill Respiratory Variables - Late Developmental Stages}

Late developmental stage tadpole brainstem preparations also exhibited the high-frequency, low-amplitude gill burst motor pattern (Table 1). Gill burst frequency $\left(\mathrm{min}^{-1}\right)$ in the late developmental stage preparations was not significantly altered by the experimental conditions. The gill burst cycle in the late developmental stage tadpoles during the transition to hypercapnia $(1.90 \pm 0.36)$ was not significantly different from control (1.84 \pm 0.34$)$; however significant gill cycle responses were observed during hypercapnia (HA), the transition to control (T2), and the final control condition (C2). In

Table 1. Gill and Lung Burst Frequency $\left(\mathrm{min}^{-1}\right)$, Cycle (sec), Duration (sec), Interburst Interval (IBI; sec), and Amplitude (au) Measured During Control (C1 \& C2), Transition to (T1) and from (T2) Hypercapnic Acidosis (HA); in Early (Stages 610) and Late (Stages 11-20) Developmental Stage Tadpoles.

\begin{tabular}{|c|c|c|c|c|c|}
\hline & C1 & T1 & HA & T2 & C2 \\
\hline \multicolumn{6}{|l|}{ Gill - Early } \\
\hline Frequency & $43.36 \pm 3.91$ & $43.84 \pm 4.61$ & $42.78 \pm 4.16$ & $45.15 \pm 4.43$ & $45.62 \pm 4.11$ \\
\hline Cycle & $1.54 \pm 0.23$ & $1.58 \pm 0.23$ & $1.57 \pm 0.19$ & $1.51 \pm 0.23$ & $1.44 \pm 0.17$ \\
\hline Duration & $1.15 \pm 0.10$ & $1.18 \pm 0.21$ & $1.17 \pm 0.12$ & $1.09 \pm 0.12$ & $1.10 \pm 0.11$ \\
\hline IBI & $0.39 \pm 0.18$ & $0.41 \pm 0.17$ & $0.38 \pm 0.12$ & $0.43 \pm 0.16$ & $0.34 \pm 0.11$ \\
\hline Amplitude & $0.17 \pm 0.02$ & $0.19 \pm 0.02$ & $0.18 \pm 0.02$ & $0.20 \pm 0.03$ & $0.18 \pm 0.02$ \\
\hline \multicolumn{6}{|l|}{ Gill - Late } \\
\hline Frequency & $39.22 \pm 5.59$ & $39.42 \pm 5.66$ & $34.60 \pm 5.59$ & $35.51 \pm 5.59$ & $38.80 \pm 4.91$ \\
\hline Cycle & $1.84 \pm 0.34$ & $1.90 \pm 0.36$ & $2.22 \pm 0.46 \dagger$ & $2.09 \pm 0.38 \dagger$ & $1.72 \pm 0.24 \dagger$ \\
\hline Duration & $1.21 \pm 0.14$ & $1.25 \pm 0.14$ & $1.25 \pm 0.14$ & $1.43 \pm 0.20 \dagger$ & $1.28 \pm 0.13 \dagger$ \\
\hline IBI & $0.58 \pm 0.22$ & $0.65 \pm 0.25 \dagger$ & $0.79 \pm 0.29 \dagger$ & $0.65 \pm 0.25 \dagger$ & $0.45 \pm 0.14$ \\
\hline Amplitude & $0.14 \pm 0.02$ & $0.15 \pm 0.02$ & $0.15 \pm 0.02$ & $0.14 \pm 0.02$ & $0.15 \pm 0.02$ \\
\hline \multicolumn{6}{|l|}{ Lung - Late } \\
\hline Frequency & $0.29 \pm 0.12$ & $1.06 \pm 0.30 \dagger$ & $0.96 \pm 0.37$ & $0.94 \pm 0.25$ & $0.63 \pm 0.26$ \\
\hline Cycle & $8.15 \pm 5.39$ & $79.97 \pm 25.88 \dagger$ & $28.61 \pm 13.32$ & $60.62 \pm 31.80 \dagger$ & $34.81 \pm 14.99$ \\
\hline Duration & $0.57 \pm 0.19$ & $1.21 \pm 0.12 \dagger$ & $0.79 \pm 0.14$ & $1.19 \pm 0.12 \dagger$ & $0.79 \pm 0.17$ \\
\hline IBI & $7.79 \pm 5.30$ & $78.86 \pm 25.83 \dagger$ & $28.03 \pm 13.19$ & $59.64 \pm 31.82$ & $34.21 \pm 14.93$ \\
\hline Amplitude & $0.19 \pm 0.07$ & $0.45 \pm 0.05 \dagger$ & $0.31 \pm 0.05$ & $0.50 \pm 0.05 \dagger$ & $0.36 \pm 0.08$ \\
\hline
\end{tabular}

†Indicates a Significantly Different Percent Change from Control (C1). 


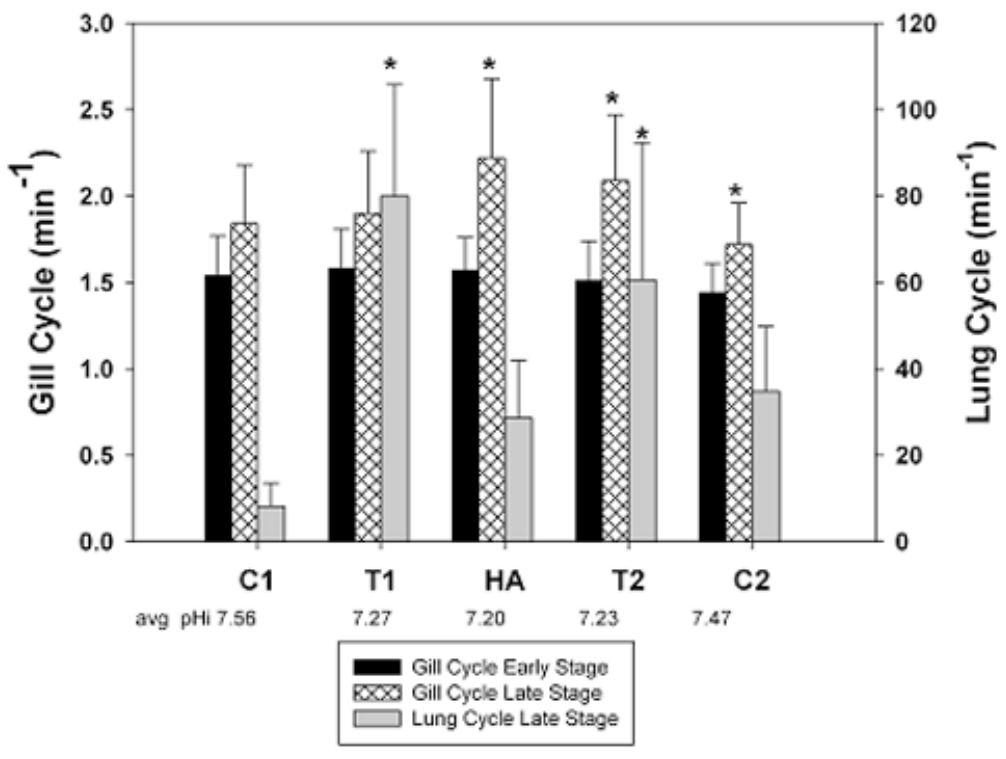

Fig. (3). Gill and lung burst cycle $\left(\mathrm{min}^{-1}\right)$ during control (C1), the transition to hypercapnia (T1), hypercapnic acidosis (HA), the transition from hypercapnia (T2) back to control bath $\mathrm{pH}$ (C2 ) for early and late developmental stage tadpoles. The average pHi in each of the five experimental conditions appears below each condition (* indicates significantly different from C1).

addition, gill burst duration during the transition to hypercapnia $(1.25 \pm 0.14)$ was not significantly different from control (1.21 \pm 0.14$)$; however gill burst duration during the transition from hypercapnia $(1.43 \pm 0.20)$ and the final control $(1.28 \pm 0.13)$ were significantly greater than control gill burst duration. Interburst interval was significantly increased in late developmental stage tadpoles during the transition to hypercapnia $(0.65 \pm 0.25)$, hypercapnic acidosis (0.79 \pm $0.29)$, and the transition from hypercapnia $(0.65 \pm 0.25)$ when compared to control $(0.58 \pm 0.22)$.

\section{Lung Respiratory Variables}

Lung activity was highly variable between animals and some early developmental stage animals did not exhibit lung bursting activity during the initial control period making it difficult to compare variables such as cycle length and IBI, as at least two events are required to calculate. On the other hand, all late developmental stage preparations exhibited the expected low frequency, high amplitude fictive lung bursting pattern. Therefore, the lung burst-related variables were examined only in the late developmental stage animals (Table 1).

Lung burst frequency $\left(\mathrm{min}^{-1}\right)$ was significantly increased during the transition to hypercapnia $(1.06 \pm 0.30)$ when compared to control $(0.29 \pm 0.12)$. The lung burst cycle was significantly increased during the transition to hypercapnia (79.97 \pm 25.88 ), the transition back to control (60.32 \pm 31.80) when compared to the initial control lung burst cycle (8.15 \pm 5.39 ). Lung burst duration (sec) during control bath $\mathrm{pH}(0.57 \pm 0.19)$ was significantly less than lung burst duration during the transition to hypercapnic acidosis (1.21 \pm $0.12)$ and the transition back to control aCSF $(1.19 \pm 0.12)$. Lung burst duration during hypercapnic acidosis $(0.79 \pm$ $0.14)$ and final control $(0.79 \pm 0.17)$ was not significantly different from any of the experimental conditions. Integrated lung burst amplitude measured in arbitrary units during transition to hypercapnic acidosis $(0.45 \pm 0.05)$ and transition back to the final control $(0.50 \pm 0.05)$ were significantly increased when compared to lung burst amplitude during control aCSF $(0.19 \pm 0.07)$. Lung burst amplitudes during hypercapnic acidosis $(0.31 \pm 0.05)$ and during the final control period $(0.36 \pm 0.08)$ were not significantly different from the initial experimental conditions.

Pearson Product Moment Correlations were performed on respiratory variables and absolute pHi unit change to determine whether there was a quantitative relationship between intracellular $\mathrm{pH}$ and the respiratory motor output. A significant $(\mathrm{P}<0.05)$ negative correlation $(-0.706)$ was obtained when comparing absolute changes in pHi from cells located in chemosensitive regions of the tadpole brainstem and lung frequency (Table 2). There was no correlation between changes in pHi and respiratory variables in cells located in non-chemosensitive regions. An example of a preparation that exhibited a negative correlation between $\mathrm{pHi}$ and lung activity is shown in Fig. (4).

\section{DISCUSSION}

Two $\mathrm{CO}_{2}$ chemosensitive regions have been identified in the tadpole brainstem [31-32]: one is rostral on the ventral surface of the brainstem at the level of $\mathrm{CN} \mathrm{V}$, and the other is more caudal, but also on the ventral surface of the brainstem at the level of CNs IX and X. In mammalian preparations, neurons in brain slices from adult animals regulate $\mathrm{pHi}$ poorly during hypercapnia whether they possess chemosensory activity or not $[6,33-34]$. When the $\mathrm{PCO}_{2}$ rises, $\mathrm{pHi}$ falls abruptly, remains low throughout the hypercapnic exposure (typically 20-40 minutes), and returns to the control level once the hypercapnic stimulus is removed. Neurons in brain slices from neonatal animals, on the other hand, regu- 
Table 2. Pearson Product Moment Correlations for Lung Respiratory Variables and Absolute pHi Unit Change in NonChemosensitive and Chemosensitive Regions of the Tadpole Brainstem

\begin{tabular}{|c|c|c|c|c|}
\hline & \multicolumn{2}{|c|}{ Non- Chemosensitive Region } & \multicolumn{2}{c|}{ Chemosensitive Region } \\
\hline & Correlation Coefficient & P Value & Correlation Coefficient & $\mathbf{- 0 . 7 0 6}$ \\
\hline \hline Frequency & 0.187 & 0.66 & 0.030 & $0.02 *$ \\
\hline Cycle & -0.090 & 0.83 & 0.443 & 0.93 \\
\hline Duration & 0.078 & 0.85 & 0.034 & 0.93 \\
\hline IBI & -0.089 & 0.83 & 0.193 \\
\hline
\end{tabular}
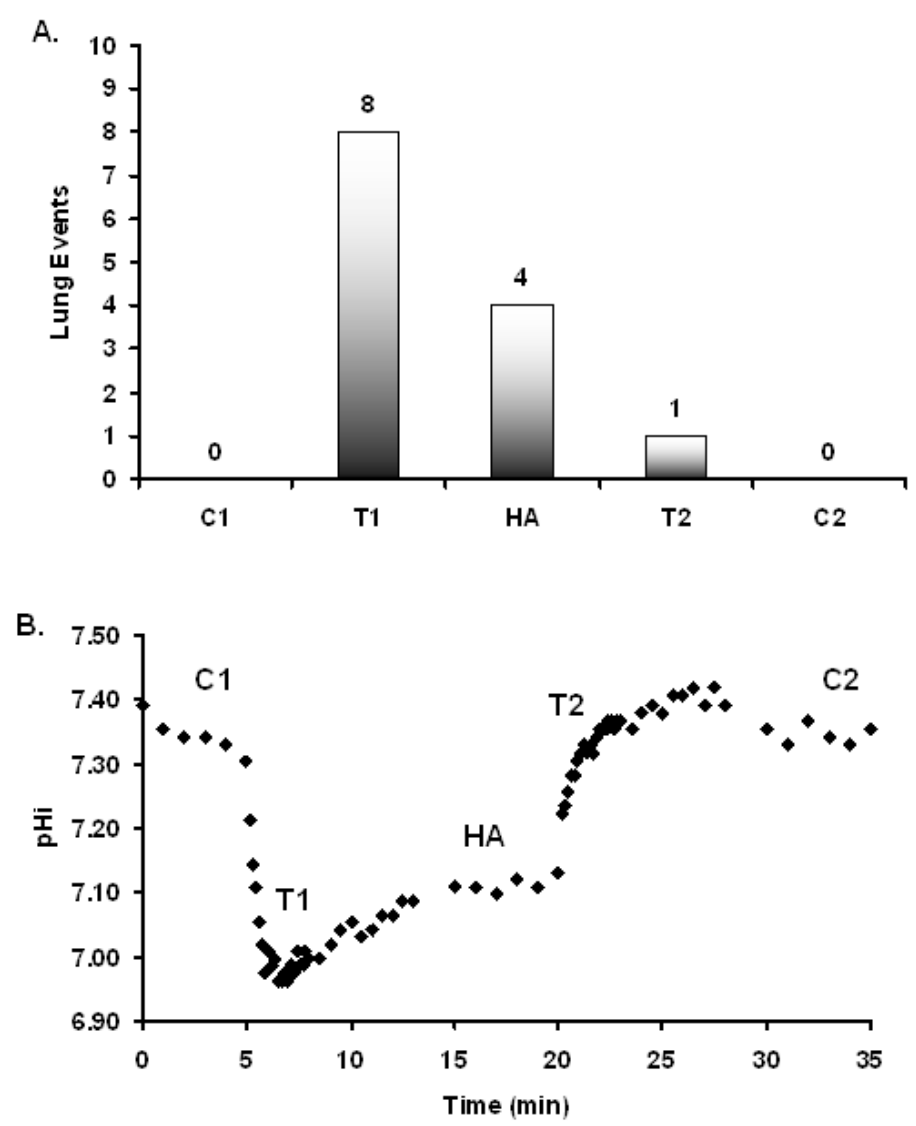

Fig. (4). Lung events expressed as a function of experimental condition (panel A) and simultaneous measurements of pHi in a single cell located in a chemosensitive region of the tadpole brainstem (panel B). Note: drops in pHi correspond to an increase in the number of lung events observed.

late $\mathrm{pHi}$ well during hypercapnia if they are nonchemosensory. The $\mathrm{pHi}$ in these neurons rises toward the control value during hypercapnia, and there is an alkaline overshoot when hypercapnia is removed as a result of the pHi regulatory processes active during hypercapnia. However, neurons in neonatal brain slices regulate $\mathrm{pHi}$ poorly (exactly like adult neurons) if they are in chemosensory nuclei $[6,34]$. All of these neurons, regardless of location and age of the animal, seem to regulate $\mathrm{pHi}$ well if $\mathrm{pHe}$ does not decrease during the acidic stress (e.g., isohydrichypercapnia). Thus, neurons possess $\mathrm{pHi}$ regulatory mechanisms, but a simultaneous decline in $\mathrm{pHi}$ and $\mathrm{pHe}$ seems to inhibit $\mathrm{pHi}$ regulation in many neurons. Therefore, we asked whether $\mathrm{pHi}$ regulation differed among chemosensory and nonchemosensory sites in the tadpole brainstem. We incubated the in vitro tadpole brainstem in $40 \mu \mathrm{M}$ of the $\mathrm{pH}$ sensitive dye BCECF-AM, washed the extracellular dye away and then used glass suction electrodes to obtain whole nerve recordings of respiratory activity while we simultaneously measured $\mathrm{pH}$ using BCECF.

We performed simultaneous recordings of spontaneous respiratory motor output and $\mathrm{pHi}$ from neurons located in known chemosensitive and non-chemosensitive regions in 
the brainstem of Lithobatescatesbeianus, an animal model well-suited to examine the neural development of respiration. Our combination of electrophysiology and fluorescence microscopy represents a novel approach to characterize central $\mathrm{CO}_{2}$ chemoreceptors in the in vitro tadpole brainstem and has resulted in several significant findings: 1 ) there are significant differences in pHi both during normocapnia and during hypercapnia between neurons located in nonchemoreceptor and chemoreceptor regions, 2) neurons located in central respiratory chemoreceptor regions demonstrate pHi recovery when only $\mathrm{pHi}$ is reduced and $\mathrm{pHe}$ is held constant, and 3) we report direct correlation between changes in pHi and respiratory motor activity unique to neurons located in chemosensory regions in a vertebrate model of respiratory control.

When exposed to hypercapnic acidosis, there was a significant difference in the ability of cells located in both chemosensitive or non-chemosensitive regions to regulate $\mathrm{pHi}$, indicating the potential for the presence of different ion transport mechanisms in cells located in chemosensitive and non-chemosensitive regions. If chemosensitive cells are to adequately monitor $\mathrm{pH}$ changes and convey these changes in $\mathrm{pHi}$ in the form of changes in firing rate, chemosensitive cells should remain relatively acidic when exposed to an acidic environment, i.e., a drop in both $\mathrm{pHo}$ and pHi. Studies in other animal preparations using $\mathrm{pH}$-sensitive probes have also identified differences in the pHi regulatory processes of cells located in chemosensitiveand non-chemosensitive regions [34-35]. In addition, using BCECF in tadpole brainstem neurons to record $\mathrm{pHi}$ optically does not disrupt respiratory rhythmicity or central respiratory chemoreception [3638]. The pHi regulatory responses to hypercapnic acidosis reported in this study are consistent with those reported in the snail [39] and rat $[6,34,35]$, indicating the evolutionary conservation of pHi regulatory mechanisms, although the persistence of $\mathrm{pHi}$ regulation in non-chemosensory cells, as seen in all tadpole developmental stages, is observed only in preparations from neonatal mammals. We observed a higher percentage of cells in non-chemosensitive regions that exhibit $\mathrm{pHi}$ regulation during hypercapnia (76\%) when compared to the cells in chemosensitive regions (47\%). Our data indicate that nearly half of the cells in chemosensitve regions regulate during hypercapnia (47\%) compared to those cells in chemosensitive regions that do not regulate $\mathrm{pHi}$ during hypercapnic acidosis (53\%); the percentage of cells exhibiting pHi regulation in the chemosensitive area in the tadpole reported in this study is similar to the percentage of neurons in the dorsal medulla of the rat (slices) that responded to hypercapnia (50\%) [40]. The percentage of neurons which respond to hypercapnia in mammals varies from approximately $20 \%$ in raphe neurons [41], to up to $80 \%$ of neurons located in the locus coeruleus [42]. It is possible that the tadpole also exhibits a developmental increase in the number of cells responding to hypercapnia as reported in rats [43] and in pre-term human infants [44]

The ammonia prepulse is a technique used to elicit changes in pHi at a constant pHo.The use of the ammonia prepulse technique in this study decreased $\mathrm{pHi}$ to a similar extent to the decreases in $\mathrm{pHi}$ achieved by hypercapnic acidosis. More importantly, the response of the cells tested with the ammonia prepulse indicated that cells which demonstrate no $\mathrm{pHi}$ regulation during $\mathrm{HA}$, a condition in which both $\mathrm{pHo}$ and $\mathrm{pHi}$ decrease, do have the ability to regulate $\mathrm{pHi}$ when only pHi drops. Together, these data suggest that the signal that initiates $\mathrm{pHi}$ regulation is strongly linked to both $\mathrm{pHo}$ and $\mathrm{pHi}$. The complement of ion transporters responsible for pHi regulation in the tadpole appear to be similar to those described in mammals. Initial studies in our lab demonstrated an adverse effect of bath applied $\mathrm{NH}_{4} \mathrm{Cl}$ on whole nerve respiratory activity; however, the disruption of respiration is not surprising, as the $\mathrm{NH}_{4} \mathrm{Cl}$ was bath applied and had the ability to alter pHi in all brainstem cells. This adverse effect has been demonstrated in other preparations [1]. Therefore other methods of focal intracellular acidification which do not disrupt central respiratory rhythmicity and chemoreception, such as uncaging $\mathrm{H}^{+}$with ultraviolet light [38], should prove beneficial in further describing the correlation between respiratory motor output and $\mathrm{pHi}$.

We recently adapted a technique to induce focal decreases in pHi alone while maintaining pHo to the tadpole brainstem preparation [38]. We bath applied the $\mathrm{H}^{+}$donor, nitrobenzaldehyde, to the tadpole brainstem and used flash photolysis to decrease only $\mathrm{pHi}$ to values similar to those observed with hypercapnia. The "uncaging" of $\mathrm{H}^{+}$using ultraviolet light induced decreases in $\mathrm{pHi}$ without disruption of respiratory rhythmogenesis. In addition, these data indicate significant $\mathrm{pHi}$ regulation following decreases in $\mathrm{pHi}$ alone similar to the rate of $\mathrm{pHi}$ regulation observed following the ammonium chloride prepulse technique reported in this study [35]. It is important to note that the lack of pHi regulation alone is not sufficient to identify a cell as a chemoreceptor, as some neurons that exhibit decreases in $\mathrm{pHi}$ and show no regulation did not show concomitant increases in firing rate [34]. Therefore, future whole cell recording confirming sustained intracellular acidification and a change in firing rate in particular neurons will be necessary.

Whole nerve respiratory activity was measured throughout hypercapnic acidosis, a condition where both $\mathrm{pHo}$ and pHi decrease. Respiratory variables were measured during five experimental conditions; control (C1), transition to hypercapnic acidosis (T1), hypercapnic acidosis (HA), transition back to control (T2), and post control (C2). It has been well documented that there is a developmental component to fictive tadpole ventilation in response of $\mathrm{CO}_{2}[31,23]$. Therefore, a subset of animals between the stages of 3 - 9 (n $=10)$ and $11-20(n=8)$ were used to describe the ontogeny of whole nerve respiratory activity through hypercapnic acidosis and return to control bath $\mathrm{pH}$.

Significant changes in the gill and lung burst timing components of cycle, duration, and IBI in response to exposure to hypercapnia were observed only in late developmental stage tadpoles, similar to findings by Taylor et al. [31] and Torgerson et al. [23]. The significant effects of hypercapnia on gill burst activities were limited to timing components, as we observed no significant changes in gill burst amplitude in response to hypercapnia in either the early or late developmental stage tadpoles. The lung burst responses to hypercapnia included both the timing components of frequency, cycle, duration, and IBI, but also included a significant increase in the lung burst amplitude. These respiratory responses to hypercapnia are similar to previously reported 
gill and lung burst responses to hypercapnia in the tadpole brainstem preparation [31, 23]. Our data support previous studies and provide new important information regarding the transition values to and from hypercapnia, periods where dynamic changes in both respiratory motor output and chemoreceptor pHi have been presumed to occur. Moreover, these findings support the contention that respiratory central chemosensory function emerges in mid to late stage tadpoles [23]. Despite equivalent reductions in pHi during hypercapnia, the early stage tadpoles were unresponsive to hypercapnia, whereas both the gill and lung bursting patterns were modified by hypercapnia in the later stage tadpoles.

In the present study there was a significant correlation between a drop in pHi in cells located in chemosensitive regions and an increase in lung burst frequency. The correlation between decreases in pHi of cells located in nonchemosensitive regions and increases in lung frequency was not observed. Because the entire brainstem was exposed to hypercapnia, it is possible that neurons other than those located in chemosensitive areas were responsible for driving ventilation. We are not claiming that the neurons located in documented chemosensitive regions that demonstrated a sustained decrease in pHi with hypercapnia are driving ventilation. Rather, these neurons in known chemosensitive regions possess one of the primary properties of central respiratory chemoreceptors, namely a maintained acidification in response to hypercapnicacidosis [42]. Although this study did not aim to record the neuronal activities of neurons, we provide evidence of two distinct populations of neurons with respect to their $\mathrm{pHi}$ profiles during hypercapnia; 1) neurons located in known non-chemosensitive areas that demonstrates regulation of $\mathrm{pHi}$ and no correlation to respiratory motor output, and 2) neurons located in previously defined chemosensitive regions that demonstrate a relative lack of regulation of pHi during hypercapnia and an inverse correlation between $\mathrm{pHi}$ and lung burst activities. The finding of a correlation between decreases in $\mathrm{pHi}$ and increases in lung frequency is novel and supports the hypothesis that decreases in $\mathrm{pHi}$ in central respiratory chemoreceptors are the proximate stimulus driving the increase in ventilation associated with hypercapnia [42] .

\section{KNOWLEDGMENT}

This research was funded by the National Institutes of Health National Center for Research Resources Research Centers in Minority Institutions (RCMI) Program grant 5G12 RR013646-08 awarded to MJG, and the National Institutes of Health National Institute of General Medical Sciences Minority Access to Research Careers (MARC) grant GM60655 awarded to DAZ.

\section{REFERENCES}

[1] Filosa JA, Dean JB, Putnam RW. Role of intracellular and extracellular $\mathrm{pH}$ in the chemosensitive response of rat locus coeruleus neurones. J Physiol 2002; 541: 493-509.

[2] Putman RW. Intracellular $\mathrm{pH}$ regulation of neurons in chemosensitive and nonchemosensitive areas of brain slices. Resp Physiol 2001;129: 37-56.

[3] Richerson GB. Serotonergic neurons as carbon dioxide sensors that maintain pH homeostasis. Nat Rev Neuro. 2004; 5: 449-61.

[4] Nattie EE, Li A. Central chemoreception in the region of the ventral respiratory group in the rat. J ApplPhysiol 1996; 81: 1987-95.
[5] Guyenet PG, Bayliss DA, Stornetta RL, Fortuna ML, Abbott SB, Depuy SD. Retrotrapezoid nucleus, respiratory chemosensitivity and breathing automaticity. Respir Physiol Neurobiol 2009; 168(12): 59-68.

[6] Ritucci NA, Chambers-Kersh L, Dean JB, Putnam RW. Intracellular $\mathrm{pH}$ regulation in neurons from chemosensitive and nonchemosensitive areas of the medulla. Am J Physiol RegulIntegr Comp Physiol1998; 275: 1152-63.

[7] Ramanantsoa N, Hirsch MR, Thoby-Brisson M, et al. Breathing without $\mathrm{CO}_{2}$ sensitivity in conditional Phox2b mutants. J Neurosci 2011; 31(36): 12880-8.

[8] Ray RS, Corcoran AE, Brust RD, et al. Impaired respiratory and body temperature control upon acute serotonergic neuron inhibition. Science 2011; 333: 637-42.

[9] Zhang X, Su J, Cui N, Gai H, Wu Z, Jiang C.The disruption of central CO2 chemosensitivity in a mouse model of Rett syndrome. Am J Physiol Cell Physiol 2011; 301(3): C729-38

[10] Brockhaus J, Ballanyi K, Smith JC, Richter DW. Microenvironment of respiratory neurons in the in vitro brainstem spinal cord of neonatal rats. J Physiol 1993; 462: 421-45.

[11] Dutschmann M, Wilson RJ, Paton JF. Respiratory activity in neonatal rats. Auton Neurosci 2000; 84:19-29.

[12] Morin-Surun MP, Denavit-Saubie M. Rhythmic discharges in the perfused isolatedbrainstem preparation of adult guinea pig. Neurosci Lett 1989; 101: 57-61.

[13] Paton JF. Functionally intact in vitro preparation generating respiratory activity in neonatal and mature mammals. Pflügers Arch1993; 428: 250-60.

[14] Richter DW, Spyer KM. Studying rhythmogenesis of breathing: comparison of in vivo and in vitro models. TRENDS Neurosci 2001; 464-72.

[15] Smith JC. Neural mechanisms generating respiratory pattern in mammalian brain stem-spinal cord in vitro. I. Spatiotemporal patterns of motor and medullary neuron activity. J Neurophysiol 1990; 64: 1149-69.

[16] Johnson SM, Turner SM, Huxtable AG, et al. Isolated in vitro brainstem-spinal cord preparations remain important tools in respiratory neurobiology. Resp Physiol Neurobiol 2012; 180: 1-7.

[17] Abdala APL, Rybak IA, Smith JC, et al. Multiple pontomedullary mechanisms of respiratory rhythmogenesis. Respir Physiol 2009; 19-25.

[18] St. John WM. Medullary regions for neurogenesis of gasping: noeud vital or noeuds vitals? J Appl Physiol 1996; 81: 1865-77.

[19] Wang W, Fung ML, Darnall RA, St John WM. Characterizations and comparisons of eupnoea and gasping in neonatal rats. J Physiol 1996; 490: 277-92.

[20] Smith JC, Abdala APLH, Koizumi H, Rybak IA, Paton JFR. Spatial and functional architecture of the mammalian brain stem respiratory network: A hierarchy of three oscillatory mechanisms. J Neurophysiol 2007; 98: 3370-87.

[21] Torgerson CS, Gdovin MJ, Kogo N, Remmers JE. Depth profiles of $\mathrm{pH}$ and $\mathrm{PO}_{2}$ in the in vitro brainstem preparation of the tadpole Rana catesbeiana. Resp Physiol 1997; 108: 205-213.

[22] Torgerson CS, Gdovin MJ, Remmers JE. Location of central chemoreceptors in the developing tadpole. Am J Physiol RegulIntegr Comp Physiol 2001; 280: 921-928.

[23] Torgerson CS, Gdovin MJ, Remmers JE. The ontogeny of central chemoreception during fictive gill and lung ventilation of an in vitrobrainstem preparation of Rana catesbeiana. J Exp Biol 1997; 299: 2063-2072.

[24] Taylor AC, Kollros J. Stages in the normal development of Rana pipiens larvae. Anat Rec 94: 7-24, 1946.

[25] Boens N, Qin W, Basaric N, Orte A, Talavera EM, Alvarez-Pez JM. Photophysics of the fluorescent $\mathrm{pH}$ indicator BCECF. J PhysChemA 2006; 110: 9334-9343.

[26] Rotman B, Papermaster BW. Membrane properties of living mammalian cells as studied by enzymatic hydrolysis of fluorogenic esters. Proc Nat AcadSci 1966; 55: 134-141.

[27] Thomas JA, Buchsbaum RN, Zimniak A, Racker E. Intracellular $\mathrm{pH}$ measurements in Ehrlich ascites tumor cells utilizing spectroscopic probes generated in situ. Biochem 1979; 81: 2210-8.

[28] Tsien RY. A non-disruptive technique for loading calcium buffers and indicators into cells. Nature 1981; 290: 527-8.

[29] Gdovin MJ, Torgerson CS, Remmers JE. Neurorespiratory patten of gill and lung ventilation in the decerebrate spontaneously breathing tadpole. Resp Physiol 1998; 113: 135-46. 
[30] Gdovin MJ, Torgerson CS, Remmers JE. Gill and lung respiratory pattern generation in the in vitro brainstem preparation of developing Rana catesbeiana. J Comp Biochem Physiol 124: 275-286: 1999.

[31] Taylor B, Harris M, Leiter JC, Gdovin MJ. Ontogeny of central $\mathrm{CO}_{2}$ chemoreception: chemosensitivity in the ventral medulla of developing bullfrogs. Amer J Physiol Regul Integr Comp Physiol 2003; 285(6): 1461-72.

[32] Taylor B, Croll AD, Drucker ML, Wilson AL. Developmental exposure to ethanol or nicotine inhibits the hypercapnicventilatory response in tadpoles. Resp Physiol Neurobiol 2008; 160: 83-90.

[33] Richerson GB. Response to $\mathrm{CO}_{2}$ of neurons in the rostral ventral medulla in vitro. J Neurophysiol1995; 73: 933-44.

[34] Ritucci NA, Erlichman JS, Leiter JC, Putnam RW. Response of membrane potential $(\mathrm{Vm})$ and intracellular $\mathrm{pH}(\mathrm{pHi})$ to hypercapnia in neurons and astrocytes from rat retrotrapezoid nucleus (RTN). Am J Physiol Regul Integr Comp Physiol 2005; 289: 85161.

[35] Nottingham S, Leiter JC, Wages P, Buhay S, Erlichman JS. Developmental changes in intracellular $\mathrm{pH}$ regulation in medullary neurons of the rat. Am J Physiol Regul Integr Comp Physiol2001; 281: 1940-51.

[36] Gdovin MJ, Zamora DA, MaruthaRavindran CR, Costanzo M, Leiter JC. Employing a pH sensitive fluorophore to measure intra- cellular $\mathrm{pH}$ in the in vitro brainstem preparation of Rana catesbeiana. Open Zool J 2009; 2: 117-23.

[37] Gdovin MJ, Zamora DA, MaruthaRavindran CR, Leiter JC. Optical recording of intracellular $\mathrm{pH}$ in respiratory chemoreceptors. Ethn Dis 2010; 20: (S1) 33-8.

[38] Ravindran MCR, Bayne JN, Bravo SC, et al. Intracellular acidosis and $\mathrm{pH}$ regulation in central respiratory chemoreceptors. J Health Care Poor Underserv 2011; 22(4): 174-86.

[39] Goldstein J, Mok JM, Simon CM, Leiter JC. Intracellular pH regulation in neurons from chemosensitive and nonchemosensitive regions of Helix aspersa. Am J Physiol Regul Integr Comp Physiol 2000; 279: 414- 23.

[40] Huang RQ, Erlichman JS, and Dean JB. Cell-cell coupling between $\mathrm{CO}_{2}$-excited neurons in the dorsal medulla oblongata. Neuroscience 1997; 80: 41-57.

[41] Wang W, Pizzonia JH, Richerson GB. Chemosensitivity of rat medullary raphe neurones in primary tissue culture. J Physiol 1998; 511: 433-50.

[42] Putnam RW. Role of pH in Cellular CO2 Chemosensitivity. J Appl Physiol 2010; 108: 1796-1802.

[43] Wang W and Richerson GB. Development of chemosensitivity of rat medullary raphe neurons. Neuroscience 1999; 90: 1001-11.

[44] Krauss AN, Klain DB, Waldman S, Auld PAM. Ventilatory response to carbon dioxide in newborn infants. Pediatr Res 1975; 9: 46-50.

(c) Zamora et al.; Licensee Bentham Open.

This is an open access article licensed under the terms of the Creative Commons Attribution Non-Commercial License (http://creativecommons.org/licenses/ by-nc/3.0/) which permits unrestricted, non-commercial use, distribution and reproduction in any medium, provided the work is properly cited. 